\title{
Le potentiel entrepreneurial dans les milieux d'enseignement
}

\author{
Louis Dussault, professeur et directeur général et Thierry Gagnon, chargé de projets \\ Centre d'entrepreneuriat et d'essaimage \\ Université du Québec à Chicoutimi
}

\section{INTRODUCTION}

Un sondage pancanadien, réalisé

récemment, révèle que $7,8 \%$ de la

population québécoise caresse l'idée de se

lancer en affaires, comparativement à

14,1 \% pour l'ensemble des Canadiens.

Depuis quelques années, les propos qui sont émis au sujet de l'entrepreneuriat au Québec ne sont guère encourageants. En effet, on évoque souvent le fait que la culture entrepreneuriale du Québec et son indice accusent un retard comparativement aux autres provinces canadiennes. À cet effet, un sondage pancanadien, réalisé récemment, révèle que $7,8 \%$ de la population québécoise caresse l'idée de se lancer en affaires, comparativement à 14,1\% pour l'ensemble des Canadiens ${ }^{1}$.

Parallèlement, dans le domaine de l'éducation, le gouvernement du Québec s'est donné comme mandat de développer l'entrepreneuriat individuel et collectif en créant, en 2004, le Défi de l'entrepreneuriat jeunesse. Pour y arriver, plusieurs mesures ont été mises en place dans les établissements d'enseignement primaire, secon- daire et de formation professionnelle afin d'y intensifier le développement de la culture entrepreneuriale.

C'est donc en lien avec ce constat que le Centre d'entrepreneuriat et d'essaimage de l'Université du Québec à Chicoutimi (CEE-UQAC) a réalisé deux études ${ }^{2}$ qui avaient comme objectif de mesurer le potentiel entrepreneurial chez la clientèle de différents niveaux scolaires (universitaire, collégial et formation professionnelle). La pertinence de réaliser de telles études réside principalement dans l'intérêt des différents intervenants socioéconomiques et du secteur de l'éducation de développer une culture entrepreneuriale régionale.

Plusieurs mesures ont été mises en place par le gouvernement du Québec dans les établissements d'enseignement primaire, secondaire et de formation professionnelle afin d'y intensifier le développement de la culture entrepreneuriale.

\section{LA THÉORIE SUR LES INTENTIONS DE DÉMARRER UNE ENTREPRISE}

En matière de cadre théorique, les deux études mentionnées ci-hauts se réfèrent à la «notion d'intention » qui est liée à la théorie du comportement planifié. Celle-ci cherche à prédire et expliquer les comportements d'un individu. On explique que la volonté d'entreprendre constitue un excellent indice de prédiction du passage à l'acte $^{3}$. Les études du CEE-UQAC se réfèrent également aux différents écrits expliquant le processus de création d'une entreprise et de l'acte de créer une entreprise ${ }^{4}$.
De plus, l'intention d'agir s'explique par des facteurs de désirabilité et de faisabilité. Ainsi, la désirabilité est liée à l'attractivité du comportement (créer son entreprise) pour l'individu alors que la faisabilité est associée aux croyances de ce dernier dans ses capacités à réaliser le projet avec succès ${ }^{5}$. Tel qu'illustré à la figure 1, le modèle indique que plus la perception de désirabilité et de faisabilité d'un projet est forte, plus l'intention de créer son entreprise est grande. 
Figure 1 : Modèle de Shapero et Sokol

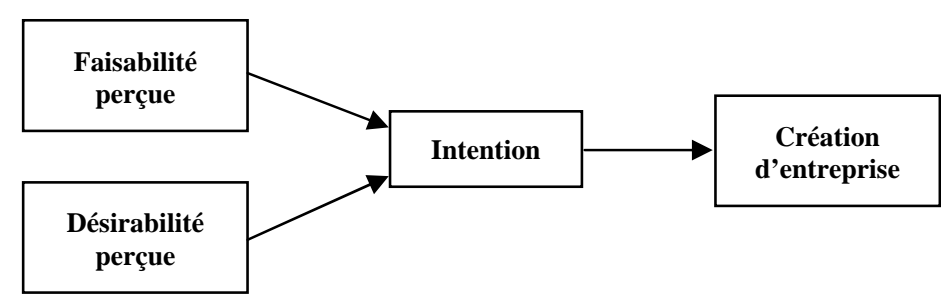

Selon l'analyse présentée ci-dessous, la création d'une entreprise s'explique par la présence d'individus possédant les caractéristiques psychologiques spécifiques. Ces individus choisissent alors de créer leur entreprise dans la mesure où ils ont identifié une occasion d'affaires et qu'ils ont fait une évaluation concrète de l'acte en termes de crédibilité et de désirabilité dans le milieu et qu'ils en perçoivent la faisabilité. Pour les fins de synthèse de cette approche théorique à la base de cette recherche, la figure 2 présente un cadre perceptuel mettant en relief le lien entre les variables sous étude.

Figure 2 : Modèle de création d'entreprise

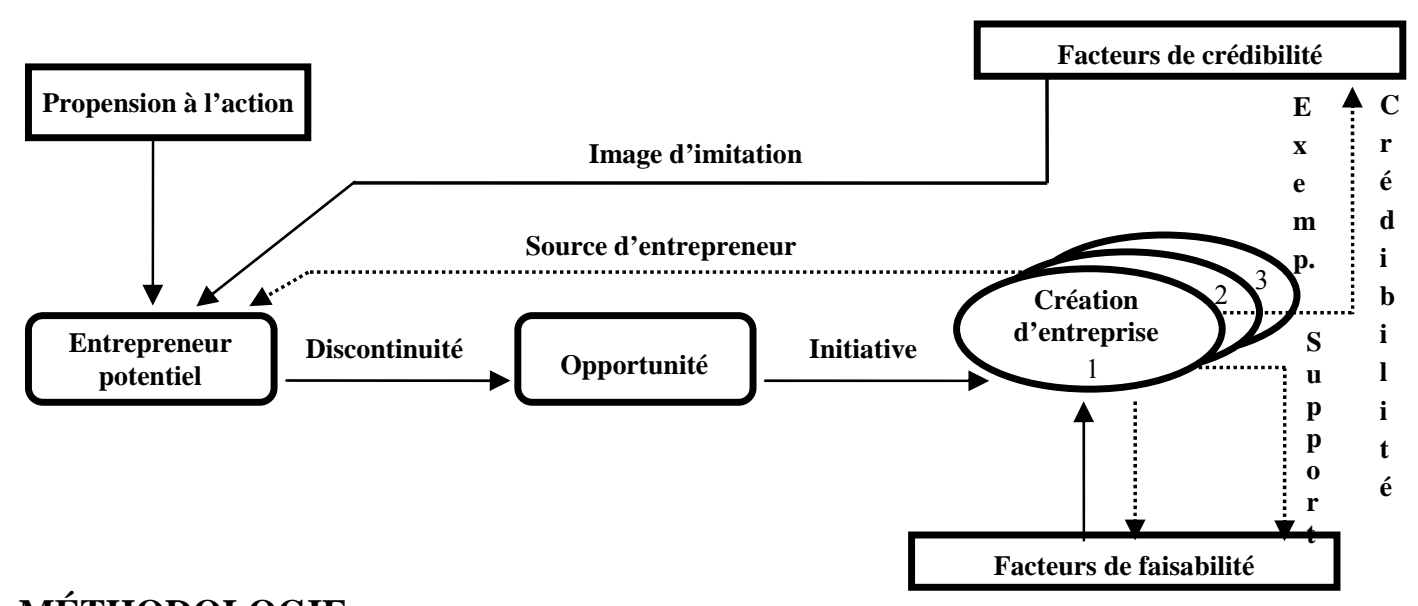

\section{LA MÉTHODOLOGIE}

Pour répondre à l'objectif principal de ces recherches, deux cueillettes de données ont été effectuées. La première a été réalisée auprès des étudiants universitaires et collégiaux du Saguenay-Lac-Saint-Jean et de Sept-Îles par l'intermédiaire d'un questionnaire en ligne. L'échantillon de cette étude était de 1408 répondants et sa marge d'erreur est de $2,55 \%$ avec un niveau de confiance de $95 \%$. La prise de données a été réalisée sur une période de 7 semaines, soit du mois de février au mois de mars 2009. La seconde étude a été effectuée auprès des élèves des centres de formation professionnelle du Saguenay-Lac-Saint-Jean à l'aide du même questionnaire et qui a été distribué en classe par les enseignants. L'échantillon de cette étude était de 1494 répondants avec une marge d'erreur de $0,0025 \%$ et un niveau de confiance de $95 \%$. La prise de données s'est échelonnée sur une durée d'environ 8 semaines, soit de septembre à octobre 2009. Le questionnaire, utilisé pour les deux études, était composé de 31 questions fermées et de quelques questions filtres. L'échelle de mesure était de type nominal et, à certaines occasions, l'utilisation d'une échelle de mesure de type ordinal et de rapport a été requise ${ }^{6}$. 


\section{ANALYSE DES RÉSULTATS}

Il est important de souligner que ces études n'ont pas été réalisées pour des fins comparatives. Cependant, les résultats enregistrés pour les différents groupes (les étudiants universitaires, les étudiants collégiaux et les élèves des centres de formation professionnelle) seront mis en évidence afin de préciser certaines dimensions.

\subsection{Le profil des répondants}

Globalement tel que mentionné au tableau 1, l'ensemble des personnes qui ont répondus au question- naire se compose de 48,6\% d'hommes et de 51,4\% de femmes. Par ailleurs, les femmes représentent la majorité des populations universitaire $(65,9 \%)$ et collégiale $(62 \%)$. Par contre, ce sont les hommes $(60,2 \%)$ qui constituent la plupart de l'échantillon pour les centres de formation professionnelle.

\section{Tableau 1 : Le profil des répondants}

\begin{tabular}{|c|c|c|c|c|c|c|c|c|}
\hline \multicolumn{9}{|c|}{ Sexe } \\
\hline & \multicolumn{2}{|c|}{ Universitaire } & \multicolumn{2}{|c|}{ Collégial } & \multicolumn{2}{|c|}{ Formation professionnelle } & \multicolumn{2}{|c|}{ Global } \\
\hline & n & $\%$ & $\mathbf{n}$ & $\%$ & $\mathbf{n}$ & $\%$ & $\mathbf{n}$ & $\%$ \\
\hline Hommes & 220 & 34,1 & 282 & 38,0 & 897 & 60,2 & 1399 & 48,6 \\
\hline Femmes & 425 & 65,9 & 461 & 62,0 & 592 & 39,8 & 1478 & 51,4 \\
\hline Total & 645 & 24,4 & 743 & 25,8 & 1489 & 50,8 & 2877 & 100,0 \\
\hline \multicolumn{9}{|c|}{ Âge } \\
\hline & \multicolumn{2}{|c|}{ Universitaire } & \multicolumn{2}{|c|}{ Collégial } & \multicolumn{2}{|c|}{ Formation professionnelle } & \multicolumn{2}{|c|}{ Global } \\
\hline & $\mathbf{n}$ & $\%$ & $\mathbf{n}$ & $\%$ & $\mathbf{n}$ & $\%$ & $\mathbf{n}$ & $\%$ \\
\hline 25 ans et moins & 432 & 66,9 & 701 & 94,2 & 1039 & 69,6 & 2172 & 75,4 \\
\hline 26 ans à 35 ans & 167 & 25,9 & 36 & 4,8 & 274 & 18,4 & 477 & 16,6 \\
\hline 36 ans et plus & 47 & 7,3 & 7 & 1,0 & 179 & 12,0 & 233 & 8,0 \\
\hline Total & 646 & 22,4 & 744 & 25,7 & 1492 & 51,8 & 2882 & 100,0 \\
\hline \multicolumn{9}{|c|}{ Statut étudiant } \\
\hline & \multicolumn{2}{|c|}{ Universitaire } & \multicolumn{2}{|c|}{ Collégial } & \multicolumn{2}{|c|}{ Formation professionnelle } & \multicolumn{2}{|c|}{ Global } \\
\hline & n & $\%$ & n & $\%$ & n & $\%$ & n & $\%$ \\
\hline Temps plein & 497 & 77,3 & 722 & 96,9 & 1461 & 98,0 & 2680 & 93,1 \\
\hline Temps partiel & 141 & 21,9 & 19 & 2,6 & 29 & 2,0 & 189 & 6,6 \\
\hline Étudiant libre & 5 & 0,8 & 3 & 0,4 & 0 & 0,0 & 8 & 0,3 \\
\hline Total & 643 & 22,4 & 744 & 25,8 & 1490 & 51,8 & 2877 & 100,0 \\
\hline \multicolumn{9}{|c|}{ Statut d'emploi } \\
\hline & \multicolumn{2}{|c|}{ Universitaire } & \multicolumn{2}{|c|}{ Collégial } & \multicolumn{2}{|c|}{ Formation professionnelle } & \multicolumn{2}{|c|}{ Global } \\
\hline & n & $\%$ & $\mathbf{n}$ & $\%$ & $\mathbf{n}$ & $\%$ & $\mathbf{n}$ & $\%$ \\
\hline Temps plein & 110 & 17,1 & 9 & 1,2 & 58 & 3,9 & 177 & 6,2 \\
\hline Temps partiel & 316 & 49,0 & 417 & 56,2 & 561 & 38,1 & 1294 & 45,2 \\
\hline Sans emploi & 207 & 32,1 & 313 & 42,2 & 827 & 56,2 & 1347 & 47,1 \\
\hline $\begin{array}{l}\text { Propriétaire- } \\
\text { copropriétaire d'une } \\
\text { entreprise }\end{array}$ & 12 & 1,9 & 3 & 0,4 & 27 & 1,8 & 42 & 1,5 \\
\hline Total & 645 & 22,4 & 742 & 25,9 & 1473 & 51,4 & 2860 & 100,0 \\
\hline
\end{tabular}

Les trois quarts des individus $(75,4 \%)$ sont âgés de 25 ans et moins et une grande partie des répondants étudient à temps plein $(93,1 \%)^{\mathrm{a}}$. De plus, un grand nombre de jeunes aux études travaillent à temps partiel. $(45,2 \%)^{\mathrm{b}}$, bien que plusieurs sont sans emploi $(47,1 \%)$.
Les étudiants collégiaux sont définitivement plus jeunes puisque $94,2 \%$ se situent dans la classe des 25 ans et moins, comparativement à $69,6 \%$ des élèves des centres de formation professionnelle et à $66,9 \%$ des étudiants universitaires. Ce sont chez les étudiants universitaires 
que l'on retrouve le plus grand nombre d'étudiants à temps partiel $(21,9 \%)$. Les élèves qui fréquentent les centres de formation professionnelle sont majoritairement sans emploi $(56,2 \%)$ alors que les étudiants universitaires $(49,0 \%)$ et collégiaux $(56,2 \%)$ travaillent à temps partiel. Les résultats obtenus reflètent les caractéristiques particulières que l'on observe dans chacune de ces populations étudiées.

\subsection{Les antécédents entrepreneuriaux des étudiants}

Les antécédents liés à la prédisposition d'un individu à l'entrepreneuriat peuvent s'expliquer par diverses variables telles que le sexe, l'âge, l'expérience de travail, le domaine d'études, l'expérience entrepreneuriale et les antécédents familiaux ${ }^{7}$. Afin d'analyser les antécédents entrepreneuriaux, nous nous sommes concentrés sur l'incidence de la présence de modèles entrepreneuriaux dans la famille et l'entourage. Ces modèles auraient une influence sur les personnes qui ont l'intention de se démarrer en affaires. Certains individus éprouvent de l'admiration envers ceux-ci et tentent de les imiter ${ }^{8}$. À la lumière des résultats du tableau 2 , on observe que ce sont les étudiants collégiaux $(2,8 \%)$ qui possèdent le moins d'expérience en affaires, comparativement aux étudiants universitaires $(6,2 \%)$ et aux élèves de la formation professionnelle $(7,0 \%)$. Par ailleurs, une quantité appréciable de l'ensemble des répondants, soit plus de $60,0 \%$, ont au moins une personne de leur entourage $^{c}$ qui est en affaires, alors que $21,8 \%$ de ceux-ci ont un membre de leur famille ${ }^{\mathrm{d}}$ en affaires.

Tableau 2 : Antécédents entrepreneuriaux

\begin{tabular}{|c|c|c|c|c|c|c|c|c|}
\hline Questions & Univ & taire & & & $\begin{array}{r}\text { For } \\
\text { profe }\end{array}$ & $\begin{array}{l}\text { ion } \\
\text { anelle }\end{array}$ & & \\
\hline $\begin{array}{l}\text { Entourage en affaires (père, mère, frère, sœur, } \\
\text { oncle, tante, grand-père, grand -mère, amis)? }\end{array}$ & $\mathbf{n}$ & $\%$ & $\mathrm{n}$ & $\%$ & n & $\%$ & $\mathbf{n}$ & $\%$ \\
\hline Oui & 411 & 63,6 & 466 & 62,9 & 897 & 60,1 & 1774 & 61,6 \\
\hline Non & 235 & 36,4 & 275 & 37,1 & 595 & 39,9 & 1105 & 38,4 \\
\hline Total & 646 & 22,4 & 741 & 25,7 & 1492 & 51,8 & 2879 & 100,0 \\
\hline Famille en affaires (père, mère)? & $\mathbf{n}$ & $\%$ & n & $\%$ & $\mathbf{n}$ & $\%$ & n & $\%$ \\
\hline Oui & 133 & 20,6 & 172 & 23,2 & 324 & 21,7 & 629 & 21,8 \\
\hline Non & 513 & 79,4 & 570 & 76,8 & 1170 & 78,3 & 2253 & 78,2 \\
\hline Total & 646 & 22,4 & 742 & 25,7 & 1494 & 51,8 & 2882 & 100,0 \\
\hline Expériences en affaires? & n & $\%$ & n & $\%$ & $\mathbf{n}$ & $\%$ & n & $\%$ \\
\hline Oui & 40 & 6,2 & 21 & 2,8 & 104 & 7,0 & 165 & 5,7 \\
\hline Non & 606 & 93,8 & 720 & 97,2 & 1390 & 93,0 & 2716 & 94,4 \\
\hline Total & 646 & 22,4 & 741 & 25,7 & 1494 & 51,9 & 2881 & 100,0 \\
\hline
\end{tabular}

Dans l'ensemble, 5,7\% de tous les répondants détiennent déjà une expérience d'affaires, c'està-dire qu'ils ont déjà possédé une entreprise. Entre autres, une analyse complémentaire, illustrée à la figure 3 , permet de constater que ce groupe de personnes a une plus forte concentration d'entrepreneurs dans leur famille $(33,1 \%)$ et dans leur entourage $(75,2 \%)$.

Une étude du Centre d'entrepreneurship HECPoly-UdeM ${ }^{9}$ indique que $82,6 \%$ des 35 lauréats de leur concours en entrepreneuriat ont un parent (père ou mère) qui a démarré une entreprise et
$80,0 \%$ de ceux-ci affirment avoir été inspirés par des modèles dans leur métier d'entrepreneur. Une autre étude, réalisée auprès de 606 jeunes entrepreneurs âgés de moins de 30 ans, démontre que $44,0 \%$ des répondants avaient un ou plusieurs entrepreneurs parmi les membres de leur famille ${ }^{10}$.

Une autre étude, réalisée auprès de 606 jeunes entrepreneurs âgés de moins de 30 ans, démontre que $44,0 \%$ des répondants avaient un ou plusieurs entrepreneurs parmi les membres de leur famille. 
Figure 3: Entourage et famille en affaires

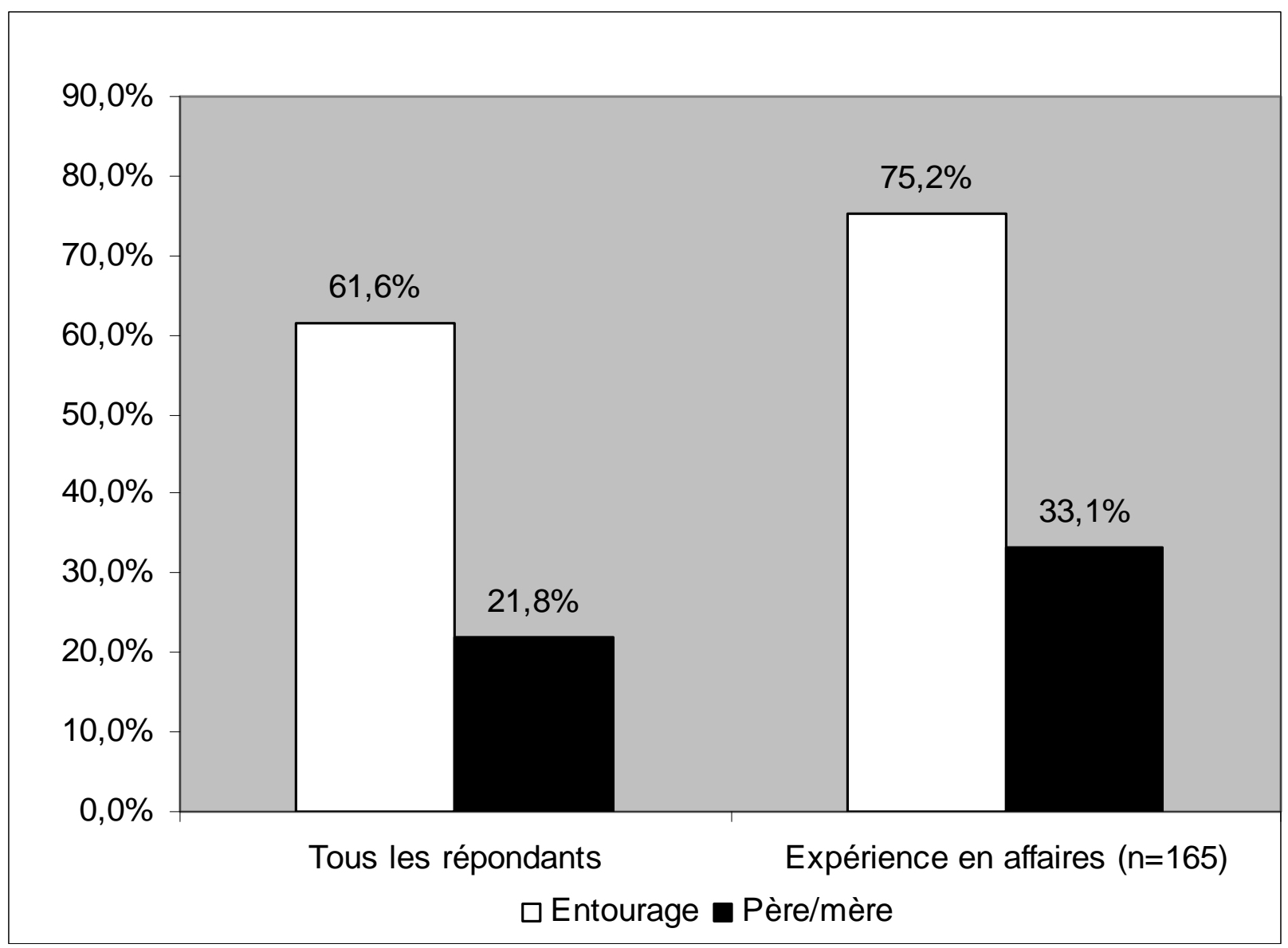

\subsection{L'idée d'affaires}

La reconnaissance d'une idée d'affaires n'est pas un élément suffisant pour qu'un individu réalise son projet d'entreprise. Il doit aussi y avoir une intention, de la part du futur entrepreneur, de vouloir passer à l'acte.

Un individu croyant détenir une bonne idée d'affaires doit la confronter avec son environnement afin de pouvoir la valider et d'y déceler une opportunité sur les marchés. L'identification et la validation d'une idée d'affaires est l'une des premières étapes dans le processus de démarrage d'entreprise. Cependant, la reconnaissance d'une idée d'affaires n'est pas un élément suffisant pour qu'un individu réalise son projet d'entreprise. Il doit aussi y avoir une intention, de la part du futur entrepreneur, de vouloir passer à l'acte ${ }^{11}$.

Comme l'indique le tableau 3, la plupart des répondants admettent avoir déjà eu une idée d'affaires (universitaire : 58,9\%; collégial : $46,7 \%$ et formation professionnelle : $45,1 \%$ ). Dans l'ensemble, cette idée émerge principalement d'un loisir ou d'un passe-temps $(18,8 \%)$, de leur domaine d'études $(24,5 \%)$, de leur expérience de travail $(19,8 \%)$ ou bien d'un champ de compétences professionnelles $(12,9 \%)$. Beaucoup ont discuté de leur idée d'affaires avec diverses personnes afin de vérifier son potentiel $(56,9 \%)$. De plus, on constate que les personnes les plus consultées font partie de ce qu'on appelle le réseau de proximité, soit les membres de la famille $(33,1 \%)$, les amis $(23,5 \%)$ et le conjoint ou la conjointe $(18,2 \%)$. 
Tableau 3 : Idée d’affaires

\begin{tabular}{|c|c|c|c|c|c|c|c|c|}
\hline \multirow{2}{*}{$\begin{array}{c}\text { Questions } \\
\text { Identification d'une idée d'affaires? }\end{array}$} & \multicolumn{2}{|c|}{ Universitaire } & \multicolumn{2}{|c|}{ Collégial } & \multicolumn{2}{|c|}{$\begin{array}{c}\text { Formation } \\
\text { professionnelle }\end{array}$} & \multicolumn{2}{|c|}{ Global } \\
\hline & $\mathbf{n}$ & $\%$ & $\mathbf{n}$ & $\%$ & $\mathbf{n}$ & $\%$ & $\mathbf{n}$ & $\%$ \\
\hline Oui & 381 & 58,9 & 348 & 46,7 & 673 & 45,1 & 1402 & 48,6 \\
\hline Non & 266 & 41,1 & 397 & 53,3 & 820 & 54,9 & 1483 & 51,4 \\
\hline Total & 647 & 22,4 & 745 & 25,8 & 1492 & 51,7 & 2886 & 100,0 \\
\hline Provenance de l'idée d'affaires? & $\mathbf{n}$ & $\%$ & $\mathbf{n}$ & $\%$ & $\mathbf{n}$ & $\%$ & $\mathbf{n}$ & $\%$ \\
\hline Domaine d'études & 177 & 29,7 & 149 & 28,5 & 241 & 20,1 & 567 & 24,5 \\
\hline Expérience de travail & 120 & 20,2 & 70 & 13,4 & 268 & 22,4 & 458 & 19,8 \\
\hline Loisir ou passe-temps & 106 & 17,8 & 138 & 26,4 & 192 & 16,0 & 436 & 18,8 \\
\hline Champ de compétences professionnelles & 74 & 12,4 & 55 & 10,5 & 169 & 14,1 & 298 & 12,9 \\
\hline Innovation/invention & 32 & 5,4 & 49 & 9,4 & 87 & 7,3 & 168 & 7,3 \\
\hline Achat d'une licence ou d'une franchise & 22 & 3,7 & 28 & 5,4 & 91 & 7,6 & 141 & 6,1 \\
\hline Offre d'un partenaire & 26 & 4,4 & 16 & 3,1 & 88 & 7,4 & 130 & 5,6 \\
\hline Observation lors d'un voyage & 23 & 3,9 & 9 & 1,7 & 39 & 3,3 & 71 & 3,1 \\
\hline Autre & 15 & 2,5 & 8 & 1,5 & 21 & 1,8 & 44 & 1,9 \\
\hline Total & 595 & 25,7 & 522 & 22,6 & 1196 & 51,7 & 2313 & 100,0 \\
\hline Personnes avec qui l'idée d'affaires a été discutée? & $\mathbf{n}$ & $\%$ & $\mathbf{n}$ & $\%$ & $\mathbf{n}$ & $\%$ & $\mathbf{n}$ & $\%$ \\
\hline Membre de la famille & 120 & 30,5 & 118 & 37,3 & 240 & 32,6 & 478 & 33,1 \\
\hline Ami & 85 & 21,6 & 90 & 28,5 & 165 & 22,4 & 340 & 23,5 \\
\hline Conjoint & 77 & 19,6 & 43 & 13,6 & 143 & 19,4 & 263 & 18,2 \\
\hline Gens d'affaires & 24 & 6,1 & 22 & 7,0 & 54 & 7,3 & 100 & 6,9 \\
\hline Collègue de travail & 35 & 8,9 & 12 & 3,8 & 33 & 4,5 & 80 & 5,5 \\
\hline Patron & 11 & 2,8 & 6 & 1,9 & 33 & 4,5 & 50 & 3,5 \\
\hline Intervenant socioéconomique & 17 & 4,3 & 7 & 2,2 & 21 & 2,9 & 45 & 3,1 \\
\hline Professeur & 7 & 1,8 & 12 & 3,8 & 22 & 3,0 & 41 & 2,8 \\
\hline Comptable & 7 & 1,8 & 2 & 0,6 & 13 & 1,8 & 22 & 1,5 \\
\hline Ingénieur & 4 & 1,0 & 1 & 0,3 & 4 & 0,5 & 9 & 0,6 \\
\hline Avocat & 1 & 0,3 & 0 & 0,0 & 3 & 0,4 & 4 & 0,3 \\
\hline Autre & 5 & 1,3 & 3 & 0,9 & 5 & 0,7 & 13 & 0,9 \\
\hline Total & 393 & 27,2 & 316 & 21,9 & 736 & 50,9 & 1445 & 100,0 \\
\hline
\end{tabular}

L'étude du Centre d'entrepreneurship HECPoly-UdeM auprès de ses 35 lauréats en entrepreneuriat indique que l'activité principale de leur entreprise est en lien avec leur champ d'études dans 68,6\% des cas. Dans cette perspective, nous pouvons donc affirmer que les entrepreneurs mettent en œuvre des projets pour lesquels ils ont de l'expérience et des connaissances. De plus, les amis et la famille joue un rôle important dans la réalisation de ces plans et, plus spécialement, lors de situations difficiles ${ }^{12}$. Les résultats des deux études confirment, eux aussi, que l'idée d'affaires provient principalement des connaissances et des expériences de travail et que la famille et les proches occupent une place importante dans le développement du projet d'affaires.

\subsection{Les intentions entrepreneuriales}

La distinction entre de fortes ou de faibles intentions entrepreneuriales d'une personne ou d'un groupe d'individus se mesure par les actions et les gestes entrepris pour mener à terme un $\operatorname{projet}^{13}$. Toutefois, il existe plusieurs causes imprévisibles qui peuvent freiner les intentions de se lancer en affaires.

Tel que présentée au tableau 4, une analyse entre les différents groupes permet de constater qu'il y a plus d'étudiants universitaires $(34,2 \%)$ et collégiaux $(35,9 \%)$ n'ayant aucun intérêt à se lancer en affaires comparativement aux élèves des centres de formation professionnelle $(23,7 \%)$. Parmi ceux qui veulent se lancer en affaires, la plupart préfèrent créer leur propre entreprise (plus de $70 \%$ dans les différents groupes) et peu d'entre eux désirent acheter une entreprise existante ou reprendre l'entreprise familiale (environ $15 \%$ pour tous les groupes). Une proportion intéressante se lancerait en affaires avec un ou des partenaires (plus de $40 \%$ pour chacun des groupes). Beaucoup de répondants ont donc l'intention de se lancer en affaires $(55,1 \%)$. Enfin, peu désirent se lancer en affaires pendant ou immédiatement après leurs études $(11,5 \%)$, mais plutôt quelques années après la fin de leurs études $(36,4 \%)$. 
Tableau 4: Les intentions de se lancer en affaires

\begin{tabular}{|c|c|c|c|c|c|c|c|c|}
\hline Questions & \multicolumn{2}{|c|}{ Universitaire } & \multicolumn{2}{|c|}{ Collégial } & \multicolumn{2}{|c|}{$\begin{array}{c}\text { Formation } \\
\text { professionnelle }\end{array}$} & \multicolumn{2}{|c|}{ Global } \\
\hline Intention de se lancer en affaires? & $\mathbf{n}$ & $\%$ & $\mathbf{n}$ & $\%$ & $\mathbf{n}$ & $\%$ & $\mathbf{n}$ & $\%$ \\
\hline Oui & 366 & 57,0 & 387 & 52,0 & 827 & 55,9 & 1580 & 55,1 \\
\hline Non & 264 & 41,1 & 344 & 46,2 & 623 & 42,1 & 1231 & 43,0 \\
\hline Je suis déjà en affaires & 12 & 1,9 & 13 & 1,7 & 29 & 2,0 & 54 & 1,9 \\
\hline Total & 642 & 22,4 & 744 & 26,0 & 1479 & 51,6 & 2865 & 100,0 \\
\hline$\frac{\text { Se lancer en affaires seul ou avec un ou des }}{\text { partenaires? }}$ & $\mathbf{n}$ & $\%$ & $\mathbf{n}$ & $\%$ & $\mathbf{n}$ & $\%$ & $\mathbf{n}$ & $\%$ \\
\hline Seul & 71 & 11,0 & 92 & 12,4 & 258 & 17,3 & 421 & 14,6 \\
\hline Avec un ou des partenaires & 272 & 42,0 & 313 & 42,1 & 689 & 46,2 & 1274 & 44,2 \\
\hline Je ne désire pas me lancer en affaires & 221 & 34,2 & 267 & 35,9 & 354 & 23,7 & 842 & 29,2 \\
\hline Je ne sais pas & 83 & 12,8 & 72 & 9,7 & 190 & 12,8 & 345 & 12,0 \\
\hline Total & 647 & 22,4 & 744 & 25,8 & 1491 & 51,7 & 2882 & 100,0 \\
\hline Manière souhaitée de se lancer en affaires? & $\mathbf{n}$ & $\%$ & $\mathbf{n}$ & $\%$ & n & $\%$ & $\mathbf{n}$ & $\%$ \\
\hline En créant mon entreprise & 313 & 74,5 & 355 & 75,1 & 849 & 75,0 & 1517 & 74,9 \\
\hline En achetant une entreprise existante & 47 & 11,2 & 50 & 10,6 & 113 & 10,0 & 210 & 10,4 \\
\hline En achetant une franchise/licence & 39 & 9,3 & 41 & 8,7 & 67 & 5,9 & 147 & 7,3 \\
\hline En reprenant l'entreprise familiale & 17 & 4,0 & 24 & 5,1 & 75 & 6,6 & 116 & 5,7 \\
\hline Autre & 4 & 1,0 & 3 & 0,6 & 28 & 2,5 & 35 & 1,7 \\
\hline Total & 420 & 20,7 & 473 & 23,4 & 1132 & 55,9 & 2025 & 100,0 \\
\hline Moment prévu de se lancer en affaires? & $\mathbf{n}$ & $\%$ & $\mathbf{n}$ & $\%$ & n & $\%$ & n & $\%$ \\
\hline Pendant mes études & 29 & 4,5 & 14 & 1,9 & 19 & 1,3 & 62 & 2,2 \\
\hline $\begin{array}{l}\text { Juste après l'obtention de mon diplôme (certificat, } \\
\text { attestation). }\end{array}$ & 48 & 7,4 & 69 & 9,3 & 150 & 10,1 & 267 & 9,3 \\
\hline $\begin{array}{l}\text { Quelques années après l'obtention de mon diplôme } \\
\text { (certificat, attestation). }\end{array}$ & 190 & 29,4 & 241 & 32,4 & 616 & 41,3 & 1047 & 36,4 \\
\hline Je suis déjà en affaires & 12 & 1,9 & 13 & 1,7 & 26 & 1,7 & 51 & 1,8 \\
\hline Je n'ai aucun intérêt & 221 & 34,3 & 267 & 35,9 & 354 & 23,7 & 842 & 29,2 \\
\hline Je ne sais pas & 145 & 22,5 & 139 & 18,7 & 327 & 21,9 & 611 & 21,2 \\
\hline Total & 645 & 22,4 & 743 & 25,8 & 1492 & 51,8 & 2880 & 100,0 \\
\hline
\end{tabular}

Une étude de la Fédération canadienne des entreprises indépendantes (FCEI) ${ }^{14}$ indique que $30,0 \%$ des gens ont déjà envisagé de posséder une entreprise alors que $23,0 \%$ y ont songé sérieusement. Toujours selon cette même étude, 43,0 \% des répondants du Québec ne souhaitent pas fonder une entreprise. Dans un autre ordre d'idées, une enquête nous informe que 7,9\% des étudiants ont pensé se lancer en affaires pendant leurs études alors que $32,2 \%$ prévoient le faire de 3 à 5 ans après leurs études ${ }^{15}$. Sommairement, les résultats de ces deux études prouvent qu'il y a une certaine concordance avec les résultats des autres recherches concernant l'entrepreneuriat, autant à l'égard de la population que dans les milieux scolaires.

\subsection{Les motivations et les obstacles à l'entrepreneuriat}

Il existe des facteurs intrinsèques qui peuvent motiver ou freiner un individu à se lancer en affaires. On peut énumérer plusieurs facteurs qui pousseraient un individu à passer à l'action. On retrouve certains éléments positifs dans les facteurs de motivation, comme le fait qu'une personne se sente prête financièrement et professionnellement ou qu'elle identifie une opportunité (nouvelle idée, nouveau marché, offre d'un partenaire, etc.). Cependant, il y a aussi des facteurs négatifs de motivation (perte d'emploi, mise à pied, insatisfaction dans son travail, mauvaises conditions de travail) ${ }^{16}$.

Il est démontré au tableau 5 que la première motivation indiquée par les répondants est "d'être son propre patron» (universitaire: $16,0 \%$; collégial : $17,9 \%$ et formation professionnelle: 23,3\%). Globalement, les autres principales motivations énoncées par les trois groupes sont: "créer mon emploi » $(15,3 \%)$, « relever des défis » $(15,1 \%)$, « la possibilité de revenus plus élevés » $(14,8 \%)$, «atteindre des objectifs personnels » $(13,7 \%)$ et « une bonne idée d'affaires » $(11,9 \%)$. 
Tableau 5: Les motivations et les obstacles à se lancer en affaires

\begin{tabular}{|c|c|c|c|c|c|c|c|c|}
\hline \multirow{2}{*}{$\begin{array}{c}\text { Questions } \\
\text { Motivation à se lancer en affaires? } \\
\end{array}$} & \multicolumn{2}{|c|}{ Universitaire } & \multicolumn{2}{|c|}{ Collégial } & \multicolumn{2}{|c|}{$\begin{array}{c}\text { Formation } \\
\text { professionnelle }\end{array}$} & \multicolumn{2}{|c|}{ Global } \\
\hline & $\mathbf{n}$ & $\%$ & $\mathbf{n}$ & $\%$ & $\mathbf{n}$ & $\%$ & $\mathbf{n}$ & $\%$ \\
\hline Être mon propre patron/indépendance & 194 & 16,0 & 253 & 17,9 & 673 & 23,3 & 1120 & 20,3 \\
\hline Créer mon emploi & 186 & 15,4 & 219 & 15,5 & 439 & 15,2 & 844 & 15,3 \\
\hline Relever des défis & 190 & 15,7 & 232 & 16,4 & 407 & 14,1 & 829 & 15,1 \\
\hline Possibilité de revenus plus élevés & 155 & 12,8 & 189 & 13,4 & 469 & 16,3 & 813 & 14,8 \\
\hline Atteindre des objectifs personnels & 174 & 14,4 & 212 & 15,0 & 371 & 12,9 & 757 & 13,7 \\
\hline Bonne idée d'affaires & 190 & 15,7 & 194 & 13,7 & 271 & 9,4 & 655 & 11,9 \\
\hline Fin d'études & 62 & 5,1 & 75 & 5,3 & 93 & 3,2 & 230 & 4,2 \\
\hline Perte d'emploi & 31 & 2,6 & 16 & 1,1 & 68 & 2,4 & 115 & 2,1 \\
\hline Tradition familiale & 24 & 2,0 & 20 & 1,4 & 58 & 2,0 & 102 & 1,9 \\
\hline Mésentente avec mon employeur & 1 & 0,1 & 4 & 0,3 & 12 & 0,4 & 17 & 0,3 \\
\hline Autre & 2 & 0,2 & 0 & 0,0 & 23 & 0,8 & 25 & 0,4 \\
\hline Total & 1209 & 22,0 & 1414 & 25,7 & 2884 & 52,4 & 5507 & 100,0 \\
\hline Obstacles à se lancer en affaires? & $\mathbf{n}$ & $\%$ & $\mathbf{n}$ & $\%$ & $\mathbf{n}$ & $\%$ & $\mathbf{n}$ & $\%$ \\
\hline Manque d'argent & 418 & 38,0 & 508 & 38,6 & 865 & 30,6 & 1791 & 34,2 \\
\hline Peur de l'échec financier & 264 & 24,0 & 364 & 27,7 & 372 & 13,1 & 1000 & 19,1 \\
\hline Manque d'expérience et de compétences & 22 & 2,0 & 16 & 1,2 & 721 & 25,5 & 759 & 14,5 \\
\hline Manque de temps & 225 & 20,5 & 235 & 17,9 & 160 & 5,7 & 620 & 11,8 \\
\hline Études non terminées & 12 & 1,1 & 36 & 2,7 & 422 & 14,9 & 470 & 9,0 \\
\hline Manque de soutien et d'aide & 73 & 6,6 & 71 & 5,4 & 134 & 4,7 & 278 & 5,3 \\
\hline Offre d'emploi & 42 & 3,8 & 18 & 1,4 & 50 & 1,8 & 110 & 2,1 \\
\hline Peur de perdre ma crédibilité & 19 & 1,7 & 33 & 2,5 & 44 & 1,6 & 96 & 1,8 \\
\hline Désaccord avec un partenaire & 6 & 0,5 & 16 & 1,2 & 29 & 1,0 & 51 & 0,9 \\
\hline Autre & 19 & 1,7 & 17 & 1,3 & 32 & 1,1 & 68 & 1,3 \\
\hline Total & 1100 & 20,1 & 1314 & 25,1 & 2829 & 54,0 & 5243 & 100,0 \\
\hline
\end{tabular}

Avant de créer sa propre entreprise, le futur entrepreneur évalue certains facteurs de l'environnement (ressources économiques, financières et sociales disponibles, milieux urbains et structures industrielles) pouvant l'influencer sur la manière de réaliser son projet d'entreprise. À ce sujet, il existe des notions de risque (financier, de carrière, familial et psychologique) qui sont associées à la réalité du démarrage d'entreprise et qui ont une influence sur les intentions d'un individu de mettre sur pied un projet d'affaires ${ }^{17}$.

Comme illustré au tableau 5, les étudiants collégiaux et universitaires ont sélectionné le manque d'argent (universitaire : $38,0 \%$ et collégial : $38,6 \%$ ), la peur de l'échec financier (universitaire : $24,0 \%$ et collégial : $27,7 \%$ ) et le manque de temps (universitaire : 20,5\% et collégial : $17,9 \%$ ) comme les principaux obstacles qui les empêcheraient de se lancer en affaires. Par ailleurs, les élèves des centres de formation professionnelle ont indiqué comme principaux obstacles au démarrage d'entreprise, le manque d'argent $(30,6 \%)$, le manque d'expérience et de compétences $(25,5 \%)$ ainsi que les études non terminées (14,9\%). D'autres études indiquent que les principales motivations des étudiants qui les inciteraient à démarrer une entreprise sont: se réaliser personnellement, relever de nouveaux défis et devenir son propre patron. Elles indiquent également que la principale raison qui les empêcherait de se lancer en affaires est le manque d'argent ${ }^{18}$.

Les élèves des centres de formation professionnelle ont indiqué comme principaux obstacles au démarrage d'entreprise, le manque d'argent, le manque d'expérience et de compétences ainsi que les études non terminées. 


\section{CONCLUSION}

\section{Les professeurs, les enseignants ainsi que les patrons ne sont pas les principales personnes avec qui l'idée d'affaires est validée, même si elle prend source dans les milieux où ces personnes sont présentes.}

L'environnement de travail et scolaire sont des milieux incubateurs propices à l'émergence d'une idée d'affaires. Toutefois, l'environnement immédiat est aussi un milieu qui favorise le développement d'idées, et ce, en raison des personnes avec qui les répondants valident ces idées (parent, conjoint et ami). Cependant, les professeurs, les enseignants ainsi que les patrons ne sont pas les principales personnes avec qui l'idée d'affaires est validée, même si elle prend source dans les milieux où ces personnes sont présentes.

Parallèlement, certains sondages indiquent que les Québécois ne semblent pas caresser l'idée de se lancer en affaires. Néanmoins, les jeunes aux études présentent certaines intentions à ce sujet. L'intérêt des jeunes envers l'entrepreneuriat doit être soutenu par des actions concrètes sachant que cette intention peut s'estomper avec le temps. La présence de modèles entrepreneuriaux joue un rôle d'influence dans le développement de la culture entrepreneuriale. Le fait qu'un intervenant démontre que l'action de créer une entreprise est réalisable peut avoir une influence positive sur les intentions du futur entrepreneur.

Plusieurs ont l'intention de se lancer en affaires avec un ou plusieurs partenaires (équipe entrepreneuriale). La majorité des étudiants souhaitent démarrer leur entreprise quelques années après l'obtention de leur diplôme. Cependant, ils sont conscients qu'ils ont un manque d'expérience, de compétences et de ressources financières pour se lancer en affaires.

Somme toute, les données recueillies permettent de mesurer les intentions entrepreneuriales des élèves et des étudiants. Elles nous indiquent qu'il existe un potentiel entrepreneurial dans les milieux scolaires. Les résultats obtenus rejoignent en grande partie la littérature et les études qui s'intéressent à la problématique de l'émergence de l'entrepreneuriat. Les intervenants socioéconomiques et du milieu scolaire peuvent s'inspirer des résultats obtenus afin d'orienter leurs actions et leurs interventions en matière de développement d'une culture entrepreneuriale plus forte.

\section{La majorité des étudiants souhaitent démarrer leur entreprise quelques années après l'obtention de leur diplôme. Cependant, ils sont conscients qu'ils ont un manque d'expérience, de compétences et de ressources financières pour se lancer en affaires.}

\section{BIBLIOGRAPHIE}

${ }^{1}$ Jolicoeur, M. Entrepreneuriat: appel à un changement stratégique, journal Les Affaires, Québec, 24 avril 2010.

${ }^{2}$ Centre d'entrepreneuriat et d'essaimage de l'Université du Québec à Chicoutimi (2009 et 2010). Le potentiel entrepreneurial des étudiants universitaires et collégiaux du Saguenay-Lac-Saint-Jean et de Sept-Îles (2009) et Le potentiel entrepreneurial des élèves des centres de formation professionnelle du Saguenay-Lac-Saint-Jean (2010).

${ }^{3}$ Ajzen, I. (1991). The theory of planned behavior. Organizational and Human Decision Processes 50, 179211; Moreau, R., Raveleau, B. (2006). Les trajectoires de l'intention entrepreneuriale, Revue Internationale PME, vol. 19, no. 2, Presses de l'Université du Québec.

${ }^{4}$ Shapero, A., Sokol, L. (1982). The social dimensions of entrepreneurship. In C. Kent, D. Sexton et K. Vesper (Éd.) The encyclopedia of entrepreneurship. Englewood Cliffs: Prentice Hall, 72-90; Belley, A. (1987). Les milieux incubateurs de l'entrepreneurship, Fondation de l'entrepreneurship, Québec.

${ }^{5}$ Shapero, A., Sokol, L. (1982). The social dimensions of entrepreneurship. In C. Kent, D. Sexton et K. Vesper (Éd.) The encyclopedia of entrepreneurship. Englewood Cliffs: Prentice Hall, 72-90; Moreau, R., Raveleau, B. (2006). Les trajectoires de l'intention entrepreneuriale, Revue Internationale PME, vol. 19, no. 2, Presses de l'Université du Québec.

${ }^{6}$ Perrien, J., Cheron, E. et Zins, M. (1983). Recherche en marketing : methods et décisions. Chicoutimi : Gaëtan Morin.

${ }^{7}$ Tremblay, M., Gasse, Y. L'impact des antécédents sur les perceptions, attitudes et intentions des étudiants collégiaux et universitaires à l'égard de l'entrepreneuriat, $\mathrm{XVI}^{\mathrm{e}}$ Conférence internationale de management stratégique, Montréal, 6-9 juin 2007. 
${ }^{8}$ Gasse, Y., D'amours, A. (1993). Profession: entrepreneur. Avez-vous le profil de l'emploi?, Fondation de l'entrepreneurship, Éditions Transcontinental Inc; Belley, A. (1990). Les milieux incubateurs de l'entrepreneurship, Fondation de l'entrepreneurship, Québec.

${ }^{9}$ Centre d'entrepreneurship HEC-Poly-UdeM. Rapport descriptif de l'étude auprès des lauréats du Centre d'entrepreneurship HEC-Poly-UdeM de 1998 à 2006, novembre 2007.

${ }^{10}$ Lorrain, J. Les jeunes entrepreneurs québécois, étude financée par le gouvernement du Québec, Université du Québec à Trois-Rivières, ministère de l'Industrie, du Commerce et de la Technologie, 1986.

${ }^{11}$ Belley, A. (1987). Les milieux incubateurs de l'entrepreneurship, Fondation de l'entrepreneurship, Québec; Gibb, A., Ritchie, J. (1982). Understanding the process of starting small businesses, European Small Business Journal, 1.1; Belley, A., Dussault, L. et Laferté, S. (2006). Comment rédiger mon plan d'affaires, $2^{\mathrm{e}}$ édition, Les Éditions Transcontinental Inc., Fondation de l'entrepreneurship; Filion, L. J. (1998). Identifier des occasions d'affaires, Revue Organisations et territoires, automne; Filion, L. J. (2007). Management des PME : de la création à la croissance, sous la direction de Louis Jacques Filion, Édition du renouveau pédagogique Inc; Laferté, S. (1998). Comment trouver son idée d'entreprise, Montréal Transcontinental.

${ }^{12}$ Fayolle, A. (2005). Introduction à l'entrepreneuriat, Paris: Dunod.

${ }^{13}$ Moreau, R., Raveleau, B. (2006). Les trajectoires de l'intention entrepreneuriale, Revue Internationale PME, vol. 19, no. 2, Presses de l'Université du Québec.

${ }^{14}$ Bruce, D., Picard, D. (2005). La relève: la clé de la réussite, Fédération canadienne de l'entreprise indépendante (FCEI).

${ }^{15}$ Gasse, Y., Tremblay, M. (2002) L'entrepreneuriat à l'Université Laval : intérêts, intention, prévalence et besoins des étudiants, Centre d'entrepreneuriat et de PME, Université Laval, automne.

${ }^{16}$ Belley, A. (1987). Les milieux incubateurs de l'entrepreneurship, Fondation de l'entrepreneurship, Québec

${ }^{17}$ Broom, H. N., Longenecker, J.G. et Moore, C. W. (1983). Small business Management, Sixth Edition, SouthWestern Publishing Co; Papin, R. (2001). Stratégie pour la création d'entreprise, 9 édition, Paris: Dunod.

${ }^{18}$ Tremblay, M., Gasse, Y. L'impact des antécédents sur les perceptions, attitudes et intentions des étudiants collégiaux et universitaires à l'égard de l'entrepreneuriat, $\mathrm{XVI}^{\mathrm{e}}$ Conférence internationale de management stratégique, Montréal, 6-9 juin 2007.

\section{Notes}

${ }^{a}$ Universitaire (étudiant inscrit à 12 crédits et plus), collégial (étudiant inscrit à au moins 4 cours), formation professionnelle (élève inscrit à au moins 180 heures d'enseignement).

${ }^{\mathrm{b}}$ Moins de 35 heures de travail par semaine.
${ }^{\mathrm{c}}$ Grand-parent, parent, frère, sœur, conjoint(e), ami(e) ou autre.

${ }^{\mathrm{d}}$ Père ou mère. 